\title{
MENTE BILINGÜE, CEREBRO BILINGÜE
}

\author{
Marta Minkiewicz \\ Universidad de Wrocław \\ http://dx.doi.org/10.18778/8220-201-4.20
}

\section{Resumen}

En el mundo actual cada vez más personas, ya sea desde la infancia o posteriormente, dominan dos o más idiomas. Gracias a los avances tencológicos los expertos pueden investigar como el bilingüismo cambia el cerebro a nivel fisiológico y como influye en las capacidades cognitivas. El objetivo de este trabajo será presentar como el bilingüismo de cerebro era percebido antes y que información nos proporcionan los últimos descubrimientos de la neurociencia y de la psicolingüística.

Palabras clave: Bilingüismo, neurolingüística, psicolingüística, capacidades cognitivas.

El fenómeno del bilingüismo ${ }^{1}$ (esto es, del domino de dos idiomas) está presente en más de la mitad de la población mundial, y en aumento -debido fundamentalmente al efecto de la globalización. Y sin embargo, pese al progreso cada vez más acelerado en los estudios sobre el cerebro en las personas bilingües todavía no conocemos en profundidad su funcionamiento. Por esta razón aún hoy bastantes especialistas -médicos, psicólogos, logopedas...- que desaconsejan a los padres que eduquen a sus hijos en el bilingüismo advirtiéndolos de los numerosos problemas que esto podría provocar.

1 Dada la extensión de este estudio no vamos a repasar aquí las definiciones de los términos bilingüe y bilingüismo; las personas interesadas pueden recurrir a literatura muy amplia. 
A lo largo del siglo XIX, y aún a principios del XX, se ha repetido una y otra vez que aprender dos idiomas simultáneamente era perjudicial para el desarrollo del niño, pues conducía no solo a la confusión entre las dos lenguas -y por tanto a la incapacidad de expresarse correctamente en una y otra- sino a trastornos en su desarrollo psicológico. Así, en 1890 el profesor Laurie, de la Universidad de Cambridge, afirmaba:

Si fuera posible que un niño viviera en dos lenguas a la vez e igualmente bien, tanto peor. Su desarrollo intelectual y espiritual no por ello se vería doblado sino reducido a la mitad. La unidad de la mente y del carácter tendrían gran dificultad en afirmarse en tales circunstancias (Baker, 1997: 157).

Uno de los estudios que más influyó en esta percepción del bilingüismo fue el llevado al cabo por Saer en 1922 y 1923. En una serie de test de inteligencia -que abarcó tanto a un grupo numeroso de niños de pequeños pueblos, como a estudiantes universitarios del País de Gales- se detectaron pocas diferencias entre los bilingües y monolingües de las ciudades, mientras que se detectó retraso cognitivo entre los niños bilingües de las poblaciones rurales. Esto llevó a este autor a afirmar que el bilingüismo no solo conducía a dos años de retraso cognitivo, sino a un desorden psicológico de por vida. Hay que tener en cuenta que esta oposición "científica" al bilingüismo se dio en países anglosajones -EE. UU., Gran Bretaña e Irlanda- en dónde se daban problemas sociopolíticos referidos a la existencia de minorías lingüísticas y culturales o de importantes masas de población emigrante. Así, por ejemplo, la obra de Saer sirvió a las autoridades británicas como argumento contra la enseñanza en galés, obviando el hecho de que en la mencionada investigación gran parte de los test se realizaron en un solo idioma (normalmente el menos usado por las personas bilingües entrevistadas) o que, como han indicado estudios posteriores, se habían dejado de lado aspectos tales como el estatus socioeconómico, las condiciones escolares, los aspectos emocionales o el nivel de conocimiento de la segunda lengua (Hoffmann, 1991: 121-123). En este sentido, la mayoría de los posteriores estudios 
o no han detectado tales retrasos cognitivos entre la población bilingüe (Darsie, 1926; Hill, 1936; Pinter y Arsenian, 1937; Arsenian, 1937; Spoerl, 1944; Darcy, 1953) o han certificado su carácter positivo (Peal y Lambert, 2007: 234-235).

En el debate en torno a los "efectos secundarios" del bilingüismo han resultado cruciales los estudios de Peal y Lambert. Esta investigación -en la que se emplearon múltiples test psicolingüísticos- tomó en consideración variables antes dejadas de lado por autores como Saer, referidas esta vez a un grupo social y culturalmente equilibrado de niños de Montreal que dominaba el inglés y el francés. Las conclusiones fueron que los niños bilingües alcanzaron resultados mucho más óptimos, tanto en las pruebas de inteligencia verbales como en las no verbales, que los monolingües, apreciándose una mayor flexibilidad mental al poseer unas capacidades intelectuales y conceptuales más diversificadas. Basándose en estos resultados se afirmó la tesis de que monolingües y bilingües diferían en su estructura intelectual (Peal y Lambert, 2007: 240-266).

Numerosos estudios parecen confirmar esta proposición. Así Bain (1974) ha observado que los bilingües equilibrados realizan mejor sus tareas a la hora de confeccionar conceptos; Torrance, Wu, Gowan y Aliotti (1970) en las referidas al pensamiento divergente y a la creatividad; Duncan y De Ávila (1979) en la confección de los tests de la independencia del campo y las pruebas de permanencia del objeto de Piaget; Ben-Zeev (1977) notó una mayor capacidad de los bilingües tanto en las pruebas verbales como en las no verbales; finalmente Ricciardelli (1992) indicó que las personas bilingües alcanzaron mejores resultados cognitivos y metalingüísticos en los test cognitivos relacionados con la creatividad y flexibilidad de pensamiento. Al respecto resultan sumamente interesantes los estudios realizados por Ellen Bialystok y sus colaboradores. Estos demuestran las mayores capacidades cognitivas de las personas bilingües en campos como el cambio de tareas, la creación de conceptos, la teoría de la mente, las matemáticas, la capacidad de concentración o la atención. No obtante, tampoco han faltado voces críticas como las de MacNab (1979), Hakuta (1987) o Reynolds (1991) (Bialystok, 2007: 271-291). 
El lenguaje es uno de los procesos cognitivos más complejos y fascinantes. Todo niño con un adecuado desarrollo lingüístico alcanza un dominio alto de su lengua materna en los primeros años de vida, lo que sugiere la existencia de "las estructuras biológicas, que nos permiten desarrollar formas particulares del lenguaje" (Guerra, 2017: 45). ¿Qué ocurre entonces con las personas que dominan dos idiomas? En el cerebro de las personas bilingües tendrían que hallarse representaciones lingüísticas de los dos idiomas. Y sin embargo aún hoy resulta muy difícil investigar neurológicamente la representación cortical de las funciones cognitivas (el lenguaje, la memoria, la emoción...) pues estas interactúan entre sí y los circuitos neuronales implicados están distribuidos en diferentes estructuras encefálicas. Hay que tener en cuenta que, hasta hace poco más de treinta años, las ideas sobre la representación cortical del lenguaje sobre las que se apoyaba la investigación del cerebro bilingüe se basaban sobre todo en la observación de personas que habían sufrido alguna lesión cerebral (Siguan, 2001: 144-145), mientras que hoy la exploración de la actividad del cerebro durante el procesamiento del lenguaje se realiza por medio de técnicas -como la resonancia magnética funcional, la tomografía por emisión de positrones o la magnetoencefalografía- que permiten medir, por ejemplo, el consumo de oxígeno o la actividad eléctrica de ciertas zonas del cerebro mientras que personas sanas llevan a cabo diversas tareas experimentales (Costa, 2017: 74). Así, aún cuando gran parte de estos estudios no indica mayores diferencias en la representación cortical de la conducta verbal entre las personas monolingües y bilingües, algunos autores sugieren que hay una serie de variables (p. ej. el nivel de conocimiento de la segunda lengua, la edad de su adquisición o la similitud entre las dos) que pueden implicar ciertas diferencias en el procesamiento de las lenguas y su representación. Pues aunque los expertos han refutado la teoría de la edad crítica como frontera tras la cual resulta imposible aprender la segunda lengua, se ha podido observar un incremento de la actividad neuronal en las regiones relacionadas con el lenguaje (el área de Broca y la ínsula) en tareas experimentales que exigen el procesamiento semántico y gramatical de una segunda lengua. 
Este fenómeno no aparece cuando ambas lenguas se han aprendido en la edad temprana y la competencia entre ellas es alta (Costa, 2017: 77). Cuando el dominio de la segunda lengua no es muy alto se observa una mayor actividad en las regiones cerebrales cercanas a las áreas clásicas del lenguaje o en las regiones vinculadas a las funciones ejecutivas, lo que confirma el concepto de eficiencia cortical de Ertl y Schafer: "cuanto mayor sea la habilidad en una tarea cognitiva específica, más eficiente será su procesamiento neuronal, es decir menor será el consumo de energía que necesita para funcionar" (Gómez-Ruiz, 2010: 446). Para explicar esta activación cerebral provocada por el procesamiento de una segunda lengua, Albert Costa indica varios elementos que no se excluyen entre sí: "el coste asociado a controlar dos lenguas, la falta de automaticidad en el procesamiento de la segunda, el esfuerzo cognitivo que esto puede conllevar y la mayor carga de control motor implicada en la utilización de esa segunda lengua" (Costa, 2017: 78).

La mayoría de las investigaciones dedicadas a los comportamientos mentales en las personas bilingües han buscado averiguar si estas disponen de dos sistemas lingüísticos independientes, o si forman un sistema único. La teoría de los sistemas independientes respalda la facilidad del bilingüe a la hora de producir, asociar o evocar las palabras en la misma lengua. Sin embargo, la habilidad de traspasar el significado de una lengua a otra sugiere un sistema único (Siguan, 2001: 148) pese que, aún hoy, se afirme que en las mentes de las personas bilingües existe algo así como un "interruptor" que les permite usar uno de los idiomas en un momento determinado, dejando en compás de espera al segundo. Así lo explicaron Kolers (1966), Macnamara y Kushnir (1971) y otros autores que hoy en día se apoyan en sus teorías (Wodniecka-Chlipalska, 2011: 264). Además muchos expertos han llegado a una solución de consenso que compagina las teorías de los dos sistemas y del sistema único, afirmando que el individuo bilingüe dispone de dos subsistemas lingüísticos vinculados en varios modos: en los aspectos perceptivos del lenguaje destaca la separación entre dos sistemas, en los niveles semánticos y conceptuales se nota más la unidad del sistema (Siguan, 2001: 148). Así según Paradis: 
... los bilingües tienen dos subsistemas de conexiones neuronales, uno para cada lengua, y cada uno puede ser activado o inhibido de forma independiente gracias a las fuertes asociaciones que existen entre los elementos de cada sistema, pero al mismo tiempo los dos subsistemas se integran en un sistema más amplio del que pueden extraer elementos de cualquiera de las dos lenguas en cualquier momento ... (Siguan, 2001: 147-148).

Hay que señalar que actualmente gran parte de las investigaciones dedicadas a analizar las interacciones entre las lenguas durante el procesamiento del habla demuestran el acceso no selectivo a ambas lenguas; esto es, que los dos idiomas están activos al mismo tiempo y que "rivalizan" entre sí, sin que el individuo se dé cuanta de esa batalla en su cabeza, sobre cuál de ellos será usado en un momento dado (Wodniecka-Chlipalska, 2011: 265-266). En palabras de Núñez:

Las dos lenguas operan con el mismo sistema central de procesamiento, no funcionan separadamente, aunque si son diferentes, como dos icebergs unidos por la base y apartados encima de la superficie. Independientemente de la lengua en que opere la persona, los pensamientos que acompañan a la conversación, la lectura, la escritura y la comprensión auditiva vienen del mismo motor central. Cuando una persona habla dos o más lenguas, hay una fuente integrada de pensamiento (Núñez, 2007: 78).

Los últimos estudios neurológicos ${ }^{2}$ también son cada vez más críticos con la idea de que existan diferencias en la representación cerebral de las dos lenguas, sobre todo cuando su conocimiento es equiparable. Así Gómez Ruiz, del Departamento de Neurología del Hospital General de L'Hospitalet, concluye:

2 Realizados entre otros por Klein et al. (1995; 1999), Chee et al. (1999), Klein et al. (1999), Hernández et al. (2000), De Bleser et al. (2003), Perani et al. (2003). 
Los bilingües o políglotas no tienen un cerebro con diferentes áreas cerebrales para cada lengua, ni regiones cerebrales exclusivas. Igualmente, las funciones cognitivas clásicamente asociadas a cada hemisferio cerebral siguen siendo las mismas en todos los individuos. (...) lo más probable es que las lenguas que habla una misma persona estén representadas como subsistemas microanatómicos distintos dentro de las mismas regiones cerebrales (Gómez-Ruiz, 2010: 450).

Las personas bilingües cuando hablan con los monolingües no suelen cometer errores ni insertan palabras o estructuras del otro idioma. Y sin embargo cuando hablan con otro bilingüe, y sobre todo cuando viven en una comunidad bilingüe, frecuentemente introducen elementos de las dos lenguas o cambian de idioma dentro de la misma frase (fenómeno que los expertos denominan cambio de código o "code-switching"). En este sentido, aunque los observadores puedan percibir este proceso como aleatorio es normalmente resultado no de un fallo lingüístico sino fruto de determinadas funciones comunicativas que suelen respetar las reglas gramaticales. Mi hijo, por ejemplo, me pide "pan con $m a-$ setko" y "agua sin bąbelki" aunque conoce la palabras "mantequilla" y "sin gas" simplemente porque en este contexto "le suenan mejor" los equivalentes polacos; cuando dice "creo que he dejado los misie en casa de los abuelos" llama la atención la concordancia del artículo determinado los con el número de la palabra en polaco. Sin embargo en un contexto monolingüe mi hijo, como otras personas bilingües, no tiende a recurrir a este tipo de "juegos" lingüísticos. Y dado que la gran parte de los estudios recientes subrayan que en el procesamiento del habla se activan las dos lenguas, y que ambas interactúan continuamente en la mente de los individuos bilingües, surge la cuestión de qué hace que estos normalmente no confundan ambos idiomas, ni inserten las expresiones de un idioma en el otro. Hasta el momento no poseemos una respuesta conclusiva. Una de las teorías que se barajan, la de Schwartz y Kroll, sugiere que, en este caso, el papel decisivo lo ejercería un mecanismo cognitivo externo al sistema lingüístico que disminuiría la capacidad de influencia del idioma que en ese 
momento no se usa, o que frenaría sus representaciones lingüísticas. Por su parte, David Green propuso un modelo de control inhibitorio en el que la clave del procesamiento del habla se referiría a la capacidad por tener bajo control la lengua que en ese momento no se utiliza. Su razonamiento lo confirman estudios empíricos como los de Meuter y Allport (1999) o de Wodniecka, Bobb, Kroll y Green (2006), que precisan que la lengua más activa -normalmente la primera- exige un mayor control inhibitorio. Esto implicaría que existe una "asimetría de costes de cambio de idioma” en la que el coste que supondría el cambio de la segunda lengua a la primera (la mejor dominada) sería mayor que el de la primera a la segunda (Wodniecka-Chlipalska, 2011:266-269).

Los especialistas intentan examinar no solo las diferencias funcionales en el cerebro de los hablantes bilingües sino también sus diferencias estructurales (o sea de la densidad de la sustancia gris y de la sustancia blanca). Las investigaciones de Andrea Mechelli y de sus colaboradores demostraron que, en las personas bilingües, el lóbulo parietal inferior del hemisferio izquierdo tenía una mayor densidad de sustancia gris que en las monolingües, tanto entre los individuos que aprendieron la segunda lengua en su infancia como más tarde. Otros estudios indican el desarrollo de la sustancia gris del putamen izquierdo, o sea en el área del cerebro responsable de la articulación y de los procesos fonológicos. También existen pruebas que muestran una mayor densidad de la sustancia gris en los ganglios basales y el tálamo (las partes más profundas de cerebro) vinculadas a la articulación, aunque hasta hace poco no se relacionase esta parte del cerebro humano con el procesamiento del lenguaje. Con respecto a los trabajos relativos a los cambios en la integridad de la sustancia blanca se dan menos estudios, aunque se han observado cambios en el fascículo occipitofrontal, en el cuerpo calloso y/o en otras fibras cerebrales (Costa, 2017: 141-146). En este sentido, tal y como mantiene Bialystok, no nos debería sorprender la presencia de la nueroplasticidad cerebral; esto es, que una experiencia intensa y continua, como puede ser el uso de dos o más idiomas, deje huellas en nuestras mentes y cerebros, y que las conexiones funcionales y las regiones estructurales aprovechadas cambien a lo largo de su uso (Bialystok, 
Craik, Luk, 2012: 247). No obstante, algunos investigadores afirman que los cambios estructurales del cerebro bilingüe son mínimos, o más bien de carácter cuantitativo que cualitativo y que, por lo tanto, no resultan significativos.

En resumen, la percepción del bilingüismo ha evolucionado de manera sustancial a lo largo de la historia: desde una visión notablemente negativa a finales del siglo XIX y en la primera mitad del siglo XX, hasta, posteriormente, un optimismo quizá, en algunos casos, excesivo. El creciente interés por el tema, el desarrollo de nuevos paradigmas experimentales y las innovaciones tecnológicas han permitido estudiar el fenómeno del bilingüismo de forma mucho más desarrollada y objetiva. La relación entre el bilingüismo y el desarrollo cerebral todavía no está lo suficientemente explorada y no podemos sacar unas conclusiones definitivas. Más bien estamos al principio de un nuevo camino, que promete ser muy largo y abierto, y que constituye una gran oportunidad a la hora de estudiar las relaciones entre las capacidades cognitivas y estructurales del cerebro humano, y el uso lingüístico monolingüe o bilingüe de los seres humanos.

\section{Referencias bibliográficas}

Baker, C. (1997), Fundamentos de educación bilingüe y bilingüis$m o$, trad. Ángel Alonso-Cortés, Madrid: Cátedra.

Bialystok, E. (2007), "Wpływ dwujęzyczności na rozwój poznawczy", trad. Zofia Wodniecka-Chlipalska, en I. Kurcz (ed.), Psychologiczne aspekty dwujęzyczności, Gdańsk: Gdańskie Wydawnictwo Psychologiczne, 269-295.

Bialystok, E., Craik, F.I.M., Luk, G. (2012), “Bilingualism: Consequences for Mind and Brain", Trends in Cognitive Sciences, $16,240-250$.

Costa, A. (2017), El cerebro bilingüe. La neurociencia del lenguaje, Barcelona: Penguin Random House Grupo Editorial.

Gómez-Ruiz, M.I. (2010), “Bilingüismo y cerebro: mito y realidad”, Neurología, 25, Barcelona, 443-452.

Guerra, E.E., (2007),“Bilingüismo: hallazgos y repercusiones metodológicas en neurociencias”, Revista chilena de neuropsicología, 2, Santiago, 44-55. 
Hoffmann, C. (1991), An introduction to bilingualism, London, New York: Longman.

Núñez, E. (2007), “Tienen ventajas cognitivas los bilingües sobre monolingües?”, Lingüística, 47, 76-85.

Peal, E. y Lambert, W.E. (2007), “Związek dwujęzyczności z inteligencją", trad. Zofia Wodniecka-Chlipalska, en I. Kurcz (ed.), Psychologiczne aspekty dwujęzyczności, Gdańsk: Gdańskie Wydawnictwo Psychologiczne, 229-268.

Siguan, M. (2001), Bilingüismo y lenguas en contacto, Madrid: Alianza Editorial.

Wodniecka-Chlipalska, Z. (2011), “Dwujęzyczność. Właściwości dwujęzycznego umysłu i specyfika badań psychologicznych nad dwujęzycznością", en I. Kurcz y H. Okuniewska (eds.), Język jako przedmiot badań psychologicznych. Psycholingwistyka ogólna i neurolingwistyka, Warszawa: Wydawnictwo Szkoły Wyższej Psychologii Społecznej “Akademica”, 253-284. 